\title{
Las mipymes mexicanas y el régimen de incorporación fiscal: un análisis de la reforma fiscal 2014
}

\section{The mexican mipymes and incorporation fiscal regime: an analysis of the 2014 tax reform}

\section{As mipymes mexicanas e oregime de incorporaçáo fiscal: Uma análise da reforma fiscal 2014}

\author{
Dra. María Lourdes López López** \\ Dra. María Consuelo González Pérez* \\ Dra. Mónica Liliana Rivera Obregón***
}

\section{RESUMEN}

El analizar la nueva imposición fiscal y los efectos de un régimen que entró en vigor en México a partir del 01 de enero de 2014, aplicado a las empresas, contrae un compromiso; colocar en el escenario las repercusiones de esta en las micros, pequeñas y medianas empresas (mipymes). Se pretende visualizar el acontecimiento de un proyecto político de incorporación de contribuyentes a la formalidad. El objetivo es analizar los mecanismos de inclusión y protección social que garanticen a los mexicanos un nivel de vida digno. El método de investigación mixto permitió conocer el sentir de los empresarios en cuanto a las nuevas disposiciones fiscales. El resultado de la investigación tiene un alto impacto en la cultura empresarial y fiscal.

Palabras clave: México, políticas públicas, régimen de incorporación fiscal, mipymes y reforma fiscal.

\section{ABSTRACT}

Analyzing the new tax rate and the effects of a regime that came into force in Mexico from January 1, 2014, applied to companies makes a commitment; put on stage the impact of this on micro, small and medium enterprises (mipymes). It is intended to display the event of a political project to incorporate taxpayers to formality. The objective is to analyze the mechanisms of inclusion and social protection guaranteeing Mexicans a decent standard of living. The mixed method research

Mexicana, Dra. en Estudios Fiscales, Maestra, Integrante del Grupo Multidisciplinario "Desarrollo Regional, MiPyMEs, Educación, Gobierno y Sociedad”, Universidad Autónoma de Sinaloa, Sinaloa, México. Correspondencia con la autora: maria.lourdes@uas.edu.mx.

** Mexicana, Dra. en Desarrollo Organizacional, Profesora investigadora de tiempo completo, Miembro del Cuerpo Académico Consolidado CAC-11: "Administración de Negocios y Desarrollo Regional”, Universidad Autónoma de Sinaloa, Sinaloa, México. Correspondencia con la autora: ponencias.ecea.uas@gmail.com.

*** Mexicana, Doctorado en Pedagogía por el CIIEN y dominio del idioma Inglés, Maestra, Integrante del Grupo Multidisciplinario "Desarrollo Regional, MiPyMEs, Educación, Gobierno y Sociedad”, Universidad Autónoma de Sinaloa, Sinaloa, México. Correspondencia con la autora: ponencias.ecea.uas@gmail.com. 
Las mipymes mexicanas y el régimen de incorporación fiscal: un análisis de la reforma fiscal 2014 /

López, et. al.

allowed to know the feel of employers about the new tax provisions. The result of research has a high impact on business and tax culture.

Keywords: México, public policy, fiscal regime incorporation, mipymes and tax reform.

\section{RESUMO}

Analisando-se a nova taxa de imposto e os efeitos de um regime que entrou em vigor no México a partir de 01 de janeiro de 2014, aplicado a empresa, contrai um compromisso; colocar no cenário as repercussóes de esta, nas micro, pequenas e médias empresas (mipymes). Pretende-se visualizar o acontecimento de um projeto político de incorporação de contribuintes para a formalidade. $\mathrm{O}$ objetivo é analisar os mecanismos de inclusão e proteção social que garanta aos mexicanos um padrão de vida digna. O método de investigação misto permitiu conhecer o sentir dos empresários sobre as novas disposiçóes fiscais. $\mathrm{O}$ resultado da pesquisa tem um grande impacto na cultura empresarial e fiscal.

Palavras-chave: México, políticas públicas, regime de incorporação fiscal, mis pipymes e reforma tributária

\section{Introducción}

En el presente año 2014, en México el tema de política pública fiscal adquiere relevancia debido a que es uno de los principales pilares de la recaudación de los ingresos que requiere la federación para sufragar los gastos públicos, y cuando se habla de acontecimientos que modifican sustancialmente la estructura fiscal de los contribuyentes se debe hacer un análisis de las repercusiones e implicaciones que traen consigo las reformas tributarias desde el punto de vista financiero en el sector empresarial y específicamente en las mipymes, considerando que se generan reformas que constituyen cambios de índole sociopolítica que enmarcan la economía del país y como consecuencia en el escenario empresarial.

La reforma fiscal que entró en vigor a partir del ejercicio fiscal 2014 no tiene como finalidad aumentar su base recaudatoria; esta nueva disposición fiscal pretende lograr que los contribuyentes se incorporen al padrón del Servicio de Administración Tributaria (SAT), en la intencionalidad de combatir la informalidad, por lo cual esta nueva imposición fiscal trae como objetivo la eliminación del régimen de pequeńos contribuyentes (REPECO) y el régimen intermedio vigentes al 31 de diciembre de 2013, consolidando estos dos en un nuevo régimen denominado Régimen de Incorporación Fiscal (RIF).

En un primer plano de este artículo se plantea la problemática de este acontecimiento de política fiscal, el cual permite dar a conocer que la fuerza económica del país está en las mipymes mexicanas, ya que representan el 99,36\% de las empresas con esas características, lo que justifica su gran participación en la aportación del ingreso para el gasto público, por lo que se considera que estas conforman el motor económico del 
país y su fortalecimiento redunda en un bienestar nacional; sin embargo, esta nueva disposición fiscal delimita su crecimiento y permanencia.

En un segundo punto se hace necesario el conocimiento de la información y conceptualización a través de la búsqueda de datos que permitió conocer la teoría respecto a las mipymes en un entorno globalizado, la cual sirvió de referente para marcar las pautas de la clasificación de las entidades empresariales, analizando además la legislación tributaria de México, un tema necesario para revisar la teoría de los impuestos vigentes.

El diseńo metodológico mixto utilizado es el que permitió la obtención de información suficiente debido a la aplicación de los instrumentos de investigación para la recopilación de la información de los sujetos que se involucran en la presente investigación; un cuestionario dirigido a los empresarios micros, pequeños y medianos y la entrevista aplicada a quienes dirigen las agrupaciones de empresarios, por lo que las técnicas aplicadas ofrecieron información necesaria para desarrollar los objetivos planteados en esta investigación.

En el cuarto punto se observa la interpretación de los datos, se muestra el marco empírico donde se analizan los datos obtenidos con la aplicación de los instrumentos de investigación; la información de este análisis se hace mediante gráficas y tablas elaboradas a partir de los datos que arrojó el programa estadístico Dyane versión 4 (diseño y análisis de encuestas), y la información obtenida en las entrevistas que ayudan a facilitar la interpretación de los datos obtenidos, mostrando así los resultados basados en el sistema que se utilizó; presentando finalmente las recomendaciones y sugerencias derivadas de la investigación que sirvan de base a los estudiosos de las ciencias sociales, a los estudiantes de posgrados y a los investigadores en la generación de nuevas líneas de investigación en materia tributaria.

Para las mipymes, el impacto de esta nueva reforma también será importante al incrementarse su base gravable, por eliminarse diversos regímenes y deducciones; específicamente en las medianas empresa, por tratarse de contribuyentes que tributan en el régimen general de Actividad empresarial y no en el RIF.

\section{Las mipymes y la reforma fiscal 2014}

En México corresponde al SAT como órgano desconcentrado de la SHCP (Secretaría de Hacienda y Crédito Público), la responsabilidad de aplicar la legislación fiscal con el fin de que las personas físicas y morales contribuyan proporcional y equitativamente al gasto público, así como de fiscalizar a los contribuyentes para que cumplan con las disposiciones tributarias y en materia aduanera, además de facilitar e incentivar el cumplimiento voluntario de dichas disposiciones, generar y proporcionar la información necesaria para el diseńo y la evaluación de la política tributaria. 
Las mipymes mexicanas y el régimen de incorporación fiscal: un análisis de la reforma fiscal 2014 /

López, et. al.

En octubre del 2013, el Poder Ejecutivo Federal presentó la propuesta de reformas fiscales a los principales ordenamientos jurídicos-tributarios, al $\mathrm{H}$. Congreso de la Unión, y dio a conocer a la opinión pública, que de ser aprobada entraría en vigor a partir del $1^{\circ}$ de enero de 2014. La exposición de motivos ${ }^{1}$, presenta el propósito de esta reforma, la cual señala la importancia que es contar con recursos que permitan, entre otros aspectos, atender proyectos sociales como: una pensión universal para personas mayores de 65 ańos y un seguro temporal de desempleo para proteger a quienes han perdido su trabajo. También se enfatizó que en el sexenio del Lic. Enrique Peña Nieto no se gravará con impuesto al valor agregado la enajenación de medicinas y alimentos, porque afectaría la capacidad de consumo de aquellos que menos tienen.

En ese sentido, dichos planes sociales pueden resultar loables en un país en el que aproximadamente la mitad de sus pobladores viven en la pobreza y muchos millones en la pobreza extrema, surgen diversos cuestionamientos: “¿quién sufragará esos programas y a costa de qué sacrificios?; ¿̧servirá esta reforma para fomentar la economía y ayudar a estos sectores marginados de la población realmente a mejorar o únicamente son paliativos?” (Ernst \& Young, 2014). Del análisis de la reforma propuesta, se observa que se trata de uno de los planteamientos más agresivos, con fines exclusivamente recaudatorios, que hemos visto en muchos años. En palabras del Secretario de Hacienda y Crédito Público (SHCP), con estas medidas se busca una recaudación adicional de 240 mil millones de pesos mexicanos ${ }^{2}$.

\section{El Régimen de Incorporación Fiscal (RIF)}

Una de las reformas fiscales significativas para 2014, fue la creación de un nuevo régimen que aplica a la mayoría de las mipymes mexicanas, denominado "Régimen de Incorporación Fiscal”. A través de este régimen, podrán incorporarse durante unos 10 años las personas físicas con poca capacidad económica y administrativa; en el cual se establece que los contribuyentes personas físicas en actividad empresarial que perciben ingresos menores a $\$ 2.000 .000,00^{3}$ podrán tributar en este nuevo régimen. Anteriormente los contribuyentes clasificados como mipymes se encontraban ubicados en el régimen de

Exposición de motivos publicada por la cámara de diputados, referente a diversas leyes modificadas, derogadas y abrogadas. En http://www.diputados.gob.mx/pef2014/ como una propuesta de iniciativas de ley que han sido aprobadas en tiempos de ser aplicadas a partir de 01 de enero de 2014.

2 Esta recaudación equivale actualmente a 17.741.375,10 dólares, al tipo de cambio de $\$ 13,52$ del día 18 de octubre de 2014.

3 Cantidad equivalente actualmente a 147.844,79 dólares, al tipo de cambio de $\$ 13,52$ del día 18 de octubre de 2014. 
pequeńos contribuyentes y régimen intermedio, mismos que estuvieron vigentes hasta el 31 de diciembre de 2014 para dar apertura al RIF a partir del 01 de enero de 2014 según su publicación en el Diario Oficial de la Federación (DOF).

Los contribuyentes que optan por el RIF deberán calcular el impuesto forma bimestral y efectuar el pago del impuesto a más tardar el día 17 del mes siguiente al bimestre que corresponda, mediante declaración que presentarán a través de los sistemas electrónicos que disponga el SAT en su página de Internet. De esta manera se espera que dichos contribuyentes logren cumplir adecuadamente con las obligaciones fiscales. La exposición de motivos de esta reforma fiscal, según Fuentes (2014), deja ver que "A través del Régimen de Incorporación se pretende que las personas físicas con actividades empresariales realicen su actividad en un esquema que les permita cumplir fácilmente con sus obligaciones tributarias. La participación en el Régimen de Incorporación traerá aparejado el acceso a servicios de seguridad social. De esta forma, se creará un punto de entrada para los negocios a la formalidad, tanto en el ámbito fiscal como en el de la seguridad social".

Dentro de este nuevo régimen también se establece una nueva modalidad de pago de los impuestos por los próximos diez años considerando una exención del pago gradual del 10\% anual, iniciando el primer ejercicio fiscal 2014 con la exoneración total del pago del ISR; para mayor comprensión se presenta el siguiente cuadro:

Cuadro 1. Reducción del pago de ISR del RIF.

\begin{tabular}{|c|c|}
\hline Periodo & Reducción de ISR \\
\hline Año 1 & $100 \%$ \\
\hline Año 2 & $90 \%$ \\
\hline Año 3 & $80 \%$ \\
\hline Año 4 & $70 \%$ \\
\hline Año 5 & $60 \%$ \\
\hline Año 6 & $50 \%$ \\
\hline Año 7 & $40 \%$ \\
\hline Año 8 & $30 \%$ \\
\hline Año 9 & $20 \%$ \\
\hline Año 10 & $10 \%$ \\
\hline
\end{tabular}

Fuente: diseño propio ${ }^{4}$.

4 Diseño propio a partir del Art. 111 penúltimo párrafo de la LISR 2014. 
Las mipymes mexicanas y el régimen de incorporación fiscal: un análisis de la reforma fiscal 2014 /

López, et. al.

Este nuevo régimen es una estrategia general del fisco, pues con estas facilidades de la reducción del pago de ISR pretende que voluntariamente más negocios se conviertan a la formalidad, en donde tendrían como beneficio pagar impuestos moderados y a su vez estar dentro de la legalidad, situación que hace recordar la aparición del régimen de Pequeńos Contribuyentes, el cual inició de la misma manera, haciéndose atractivo para el contribuyente y metiéndolos dentro de la formalidad. Situación que no funcionó del todo, como era esperado por Hacienda, pues en la actualidad se tuvo que abrogar dicho régimen.

\section{El contexto de las mipymes mexicanas}

De acuerdo con el Sistema de Información Empresarial Mexicano (SIEM, 2014), enuncia que en nuestro país, de la totalidad de las empresas registradas actualmente el 92,67\% son micro; $5,66 \%$ son pequeñas; $1,33 \%$ son medianas y solamente el $0,64 \%$ se clasifican como grandes. De esta manera, se tiene que en México el 99,36\% de las empresas son micro, pequeñas y medianas, lo que justifica su gran participación en la aportación del ingreso para el gasto público, por lo que estas empresas conforman el aporte económico principal del país en lo que se refiere a la recaudación en la formalidad y su fortalecimiento influye en un bienestar nacional; sin embargo, el nuevo régimen fiscal de alguna manera delimita su crecimiento y permanencia, ya que las nuevas formas de tributar establecidas cambian la estructura fiscal en el cumplimento de sus obligaciones tributarias.

Las mipymes son factores estratégicos para la estabilidad social y económica del país, ya que son las que generan mayor número de empleos, incluso más que las grandes compañías. En estas últimas fechas, se ha creado un gran interés por parte de los diferentes grupos económicos.

La Ley para el Desarrollo de la Competitividad de la Micro, Pequeńa y Mediana Empresa en México (LDCMPME), en su artículo $3^{\circ}$ fracción III, declara que las mipymes estarán legalmente constituidas, con base en la estratificación establecida por la Secretaría de Economía (SE), de común acuerdo con la SHCP y publicada en el DOF en su última modificación del 18 de enero de 2012, partiendo del número de trabajadores, quedando como sigue:

Tabla No. 3. Tamaño de la empresa por número de trabajadores.

\begin{tabular}{|c|c|c|c|}
\hline Sector/Tamaño & Industria & Comercio & Servicios \\
\hline Micro & $0-10$ & $0-10$ & $0-10$ \\
\hline Pequeña & $11-50$ & $11-30$ & $11-50$ \\
\hline Mediana & $51-250$ & $31-100$ & $51-100$ \\
\hline
\end{tabular}

Fuente: diseńo propio a partir de LDCMPME Art. $3^{\circ}$ fracc. III. 
La LDCMPME en el mismo artículo fracción IX, considera a mipymes como agrupamientos empresariales.

La importancia de las mipymes radica en la cantidad de establecimientos de este tipo que existen en nuestro país; sin embargo, estas se han enfrentado en múltiples ocasiones a escenarios adversos, en los cuales es difícil la supervivencia; ante un entorno económico desfavorable, hasta ocho de diez empresas cierran en los dos ańos de haber comenzado a operar.

De acuerdo a los estudios realizados por México Emprende 5 (2011), las microempresas son un motor de crecimiento económico y de empleo fundamental para el país, ya que, de acuerdo a los resultados del último censo económico que presenta el Instituto Nacional de Estadística y Geografía (INEGI); de cada 100 empresas mexicanas, 96 son microempresas, contribuyen con el 40,6\% del empleo y aportan el 15\% del producto interno bruto (PIB).

También presenta las desventajas a las que se enfrentan estas empresas, tomando en cuenta la competitividad y productividad de las microempresas, sobre todo de las de tipo tradicional que están siendo amenazadas por la incorporación de modernos conceptos de negocio, que evidencian: limitada profesionalización, crecimiento desordenado, rezago tecnológico, altos consumos de energía, imagen comercial descuidada e insalubre, Administración informal (“de cajón”) y limitados accesos al financiamiento.

De igual manera Araujo (2008), dice que en México las microempresas se han visto severamente impactadas por el Sistema Tributario Mexicano (STM), trayendo esto serios problemas para su permanencia en el mercado y, en el peor de los casos, provocan el cierre masivo de negocios que no han logrado resistir, corresponder o adaptarse a toda esta serie de cambios y movimientos que se han dado en el entorno que los rodea. Es importante reconocer que las mipymes prácticamente no cuentan con apoyos fiscales, a pesar de que son generadoras de empleo y que el 95,86\% de estas sostienen la economía del país.

\section{Formalidad e informalidad de las empresas}

De acuerdo con los estudios realizados por la Organización Mundial de Comercio (OMC) y la Organización Internacional del Trabajo (OIT), se establece que los efectos adversos de la informalidad están relacionados principalmente con la ausencia de au-

5 http://www.mexicoemprende.com.mx/ MéxicoEmprende es un Blog y Comunidad Online que ayuda a emprendedores a crear, administrar y hacer crecer negocios exitosos. 
Las mipymes mexicanas y el régimen de incorporación fiscal: un análisis de la reforma fiscal 2014 /

López, et. al.

mentos de la productividad y con el reducido tamaño medio de las empresas resultantes de los obstáculos al crecimiento de las empresas en la economía informal.

La capacidad empresarial y la inclinación a emprender actividades que entrańan riesgo se reducen cuando hay un alto nivel de informalidad, en parte como resultado de sistemas de impuestos mal diseñados, una protección social inadecuada y una reglamentación comercial deficiente, OMC (2009). Los análisis del efecto de la apertura del comercio en el tamańo de la economía informal, sugieren que depende fundamentalmente de las circunstancias específicas de los países y del diseńo de las políticas comerciales e internas.

La reducción de la informalidad puede hacer que surjan nuevas fuerzas productivas, aumentar la diversificación y reforzar la capacidad de participar en el comercio internacional. Los efectos adversos de la informalidad pueden estar relacionados principalmente con la ausencia de aumentos de la productividad y con el reducido tamańo medio de las empresas resultantes de los obstáculos al crecimiento de las empresas en la economía informal. La capacidad empresarial y la inclinación a emprender actividades que entrańan riesgo se reducen cuando hay un alto nivel de informalidad, en parte como resultado de los sistemas fiscales mal diseñados, la escasa protección social y la reglamentación deficiente de las empresas. La informalidad impide también que los países se beneficien plenamente de la reforma del comercio al crear trampas de pobreza para los trabajadores en transición entre empleos.

En consecuencia, cuanto mayor es la incidencia de informalidad, mayor es la vulnerabilidad de los países en desarrollo a las conmociones como la actual crisis económica mundial; por el contrario, los países con grandes economías informales sufren más frecuentemente conmociones y tienen tasas de crecimiento sostenible más bajas. En la teoría de De Soto (1989), la elección entre formalidad o informalidad puede presentarse como una decisión racional; las unidades económicas sopesan los beneficios y los costos de la formalización y consideran sus propias dificultades institucionales y de recursos. Los costos de la formalización pueden dividirse en costos de acceso a la economía formal y costos de permanencia en la misma.

En este tenor, Loayza (1996) manifiesta que los costos de acceso a la legalidad son elevados en América Latina, y que la permanencia en el sector formal puede ser muy costosa; de igual forma, las tasas impositivas marginales aplicadas a las empresas formales suelen ser muy elevadas en los países en desarrollo, en razón de la estrechez de la base impositiva. Por último, este autor señala que los requisitos burocráticos representan otro costo importante de la permanencia en el sector formal.

Considerando lo anteriormente expuesto, la nueva reforma fiscal busca integrar a la informalidad proporcionando las herramientas de crecimiento y permanencia, ya que 
por este medio permitirá a los empresarios aprovechar los beneficios de seguridad social que alcanzaría con el apego a este nuevo régimen de incorporación, esto de acuerdo a la exposición de motivos de la reforma fiscal 2014.

\section{Descripción del método de investigación}

Esta investigación, cuya finalidad es el análisis o experimentación de situaciones para el descubrimiento de nuevos hechos, la revisión o establecimiento de teorías y las aplicaciones prácticas de las mismas, se basa en los principios de observación y razonamiento y necesita en su carácter científico el análisis técnico de datos para obtener de ellos información confiable y oportuna (Chandler, 2000). Definir los enfoques metodológicos que sirven para llevar a cabo la investigación, considerando a esta como un proceso constituido por diversas etapas, pasos o fases, interconectadas de una manera lógica y dinámica. Por lo cual se concibe al método mixto como el tipo de investigación que se utilizó para llevar a cabo dicha investigación.

El método mixto se concibe como un proceso mediante el cual se recolectan, analizan y relacionan datos obtenidos tanto en investigaciones cualitativas como cuantitativas, pretendiendo obtener una respuesta a las interrogantes del planteamiento de un problema, según Rodríguez (2010). Por esto los instrumentos metodológicos se pueden considerar como las técnicas específicas de investigación, las gráficas de los resultados del cuestionario como instrumento de investigación y las correlacionadas estadísticas de las mismas consideradas cuantitativas, así como también técnicas cualitativas que son observación, entrevistas y grabaciones en video o audio; igualmente se puede afirmar que estas técnicas sólo son más o menos útiles en términos de la teoría y de la metodología utilizada y de las hipótesis que quieran ser verificadas.

Para el presente trabajo se utilizó el enfoque mixto, en virtud de que ambos se entremezclan en la mayoría de sus etapas, por lo que es conveniente combinarlos para obtener información que permita triangularla.

Para cumplir con el objetivo de esta investigación, durante los meses de julio y agosto del ejercicio 2014, se procedió a seleccionar las empresas de manera aleatoria, la cantidad de 110 negocios de las características de las mipymes, partiendo del análisis de los directorios proporcionados por la Cámara Nacional de Comercio (CANACO), la Cámara Nacional de la Industria de Restaurantes y Alimentos Condimentados (CANIRAC), así como también, por la Cámara Nacional de la Industria y la Transformación (CANACINTRA) y de la cantidad de las micros y pequeńas empresas del sector empresarial registradas en el Directorio Estadístico Nacional de Unidades Económicas (DENUE) por INEGI (2014), llegando a la conclusión de que con los datos de este 
Las mipymes mexicanas y el régimen de incorporación fiscal: un análisis de la reforma fiscal 2014 /

López, et. al.

último, se determina la base considerando del padrón registrado en el DENUE un universo de 843 empresas del sector antes mencionado.

Tomando en cuenta que el trabajo de campo es un proceso metodológico donde se elaboran los instrumentos que se aplicarán a los sujetos de la investigación, se procedió a diseñar los cuestionarios, que se aplicaron de manera presencial eligiendo a los informantes primarios que son los propietarios de las empresas ubicadas en el cuadro central de la ciudad de Guasave, Sinaloa; en los que se incluyeron preguntas necesarias relacionadas con la formalidad de la empresa, su permanencia en el mercado y sus efectos financiaros a través del cumplimento de sus obligaciones fiscales, específicamente las mipymes incorporadas en el RIF.

Para procesar la información recabada con la aplicación de la técnica del cuestionario a los empresarios, se utilizó el software Dyane 4.0 que significa: Diseño y análisis de encuestas en investigación social y de mercados; su uso consistió en la combinación de un libro y un programa informático, aplicado por el sistema Windows, que tienen la finalidad común de que el lector o usuario de los mismos aprenda y pueda hacer investigación social y de mercados.

El programa está estructurado de forma modular, con menús que facilitan el acceso a las distintas funciones y técnicas que incluye. En este se pueden diseñar cuestionarios, grabar datos y aplicar técnicas estadísticas de análisis de datos. También se pueden realizar gráficos, con el fin de mejorar la presentación de los resultados (Santesmases, 2010). Tanto los datos como los resultados y gráficos de los análisis se pueden imprimir, exportar a otros programas o guardar en ficheros para ser tratados en procesadores de texto y hojas de cálculo.

En definitiva, Dyane 4.0 es una herramienta de análisis, interactiva, fácil de aprender y sencilla de utilizar, que hace ameno y gratificante el proceso de diseño de la investigación y el análisis estadístico de los datos, y considerando que todos los sujetos de investigación poseen el atributo para ser cuestionados, ya que son de fácil como son los micro y pequeños empresarios y demás actores involucrados en la misma, de los cuales se obtuvo su percepción acerca de la efectos que ocasiona la nueva reforma fiscal.

\section{Datos recopilados y análisis de resultados}

El escenario de la investigación se centra en las actividades empresariales que marcan los índices del desarrollo económico de un país, ya que la mipymes son las que representan el mercado comercial, industrial y de servicios. Por lo tanto, las características de la empresa involucran el sentido humano y la tendencia al campo de los negocios y con ello el conocimiento para dinamizarlo. 
Para conocer el sentir y pensar del empresario se aplicó una serie de cuestionamientos, que nos llevan a entender a estas figuras empresariales, que arribaron a la actividad como empresas familiares y que evolucionan para lograr obtener incremento en sus utilidades, por lo tanto, se necesita un impulso a innovaciones técnicas y administrativas. Por otra parte, la identidad como factor hacia el ámbito empresarial expresado como crecimiento personal de un empresario que crea y recrea nuevas formas de impulso empresarial. La identidad se presenta en primer nivel como el lenguaje de realización, basado en la permanencia y éxito.

Los cuestionamientos presentados a los sujetos de esta investigación, van direccionados hacia la satisfacción que representa a los empresarios el pertenecer a un nuevo régimen fiscal que en sus objetivos principales de este, está la formalidad ante las autoridades fiscales y fomentar el crecimiento y permanencia de las mipymes; algunos de estos cuestionamientos son:

- ¿En qué régimen fiscal está registrada su empresa?

- ¿Qué tipo de registros utiliza en su negocio?

- Pagos que realiza (impuestos)

- Pagos que realiza (seguridad social)

- Servicios que paga

- ¿Usted lleva su propia contabilidad?

- ¿Utiliza los servicios externos de un profesional de la contaduría?

- ¿Qué tipo de información fiscal presenta?

- Agrupaciones a las que pertenece el empresario

- ¿Recibe capacitación por parte de la agrupación empresarial para cumplir con sus obligaciones fiscales?

- ¿Está de acuerdo con la medida que el gobierno aplica para obligar a los contribuyentes a permanecer en el RIF?

- ¿Está informado de los requisitos para pertenecer al régimen de incorporación fiscal?

- ¿Cuenta con las herramientas tecnológicas para llevar a cabo las actividades fiscales que requiere el régimen?

- ¿Cuáles son las herramientas tecnológicas con las que cuenta?

- ¿La información que proporciona el SAT ha sido de utilidad para su empresa?

- ¿Conoce los beneficios del RIF?

- ¿Ha aplicado estrategias fiscales para reducir los efectos derivados de las reformas fiscales al pertenecer a este régimen fiscal?

- ¿Lleva a cabo la planeación de sus pagos de impuestos? 
Las mipymes mexicanas y el régimen de incorporación fiscal: un análisis de la reforma fiscal 2014 / López, et. al.

- ¿Cumple en tiempo y forma con sus obligaciones fiscales?

- ¿Le ha causado algún problema la nueva reforma fiscal, en su permanencia como empresario?

De acuerdo a la aplicación del cuestionario y las entrevistas se determina el análisis de la reforma fiscal mexicana de 2014; específicamente en lo que se refiere a las mipymes, se presenta a continuación la representación gráfica de los resultados.

Gráfica 1. Razones para iniciar el negocio.

La pregunta planteada fue ¿Cuáles son las principales razones para iniciar el negocio? a lo que los empresarios, respondieron que en sus inicios como tales, predomina mayormente la actividad empresarial como el seguimiento de realización personal $(38,8 \%)$, sin embargo este tipo de empresas también genera el seguimiento de una tradición familiar $(22,4 \%)$. Esta gráfica muestra la identidad del empresario y lo dinámico, entendiendo esto como el factor que permite impulsar la existencia y convivencia de la empresa: desde lo hereditario, como lo es una empresa familiar; el cómo incrementó sus ingresos, así como la realización personal. Estas características de menor a mayor grado de importancia, proyectan que esta actividad tiene un proceso dinámico que conlleva a la realización a través de la acción innovadora de ser y estar en el ámbito empresarial.

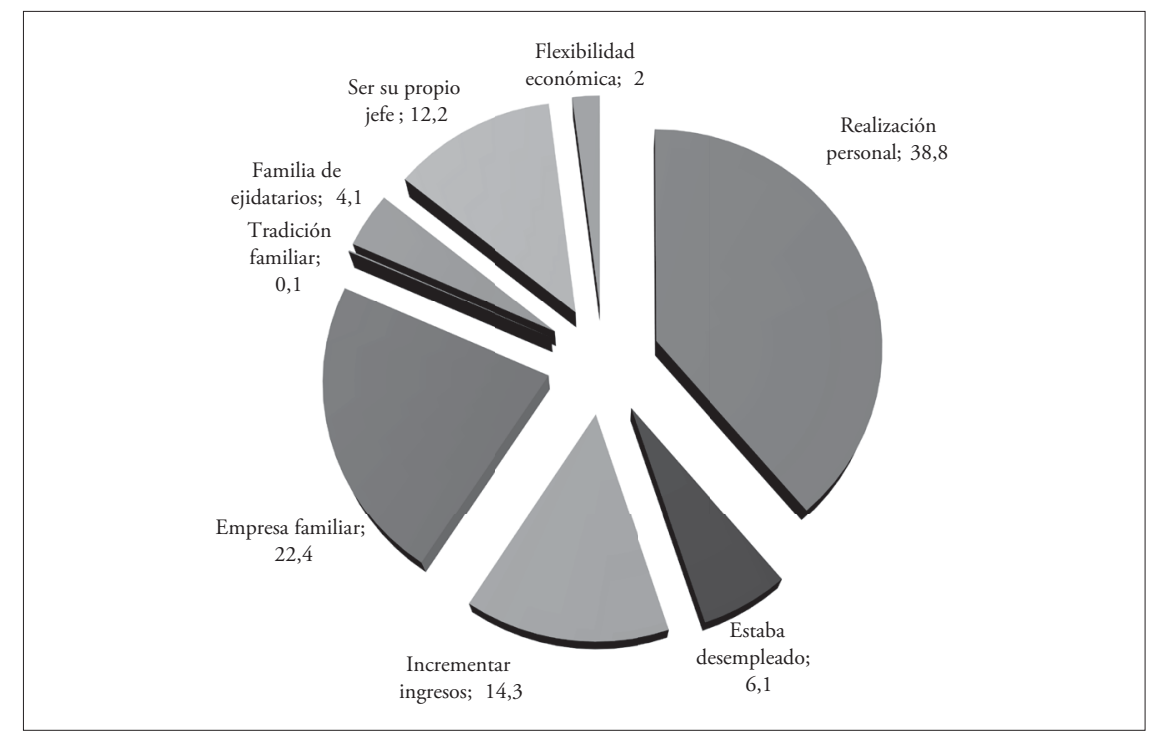

Fuente: diseńo propio a partir de la aplicación del cuestionario

A su vez, la intencionalidad del empresario adquiere sentido en el proceso de transformaciones del campo a la ciudad, las distancias se acercan; la ruralidad y la urbanidad 
adquieren orientación vinculada en cada paso de su producción; la ciudad se transforma en componente del mercado de la ruralidad y esta última se adentra en la ciudad. Este es el caso del surgimiento del ejidatario en el sector empresarial $(4,10 \%)$ en su nuevo papel; en sí ese nuevo rol hace emerger un factor innovador en la línea productiva. Esto se interpreta como evolución de perspectivas de desarrollo de los espacios micro y pequeño; se puede decir que se genera un empresario en correspondencia a su comunidad (entorno pequeño, rancho).

Gráfica 2. Régimen fiscal de tributación de la empresa.

El empresario se ubica en la clasificación de micro y pequeño empresario, debido a que la actividad generada de un ámbito empresarial familiar, innovador, de permanencia y crecimiento, le permite permanecer en el estatus del RIF, en un 68,7\%, mientras que la informalidad se manifiesta en gran medida, en 4,2\%, misma que representa una inestabilidad en su permanencia empresarial; así mismo las empresas que manejan un régimen general están en un 27,1\%, tomando en cuenta que el instrumento fue aplicado a 50 empresarios. Respecto a la importancia del cumplimiento de obligaciones fiscales, el empresario refiere un contexto de civilidad, ya que en su andar fiscal pretende establecer un vínculo formal con las autoridades fiscales y en su propia administración empresarial.

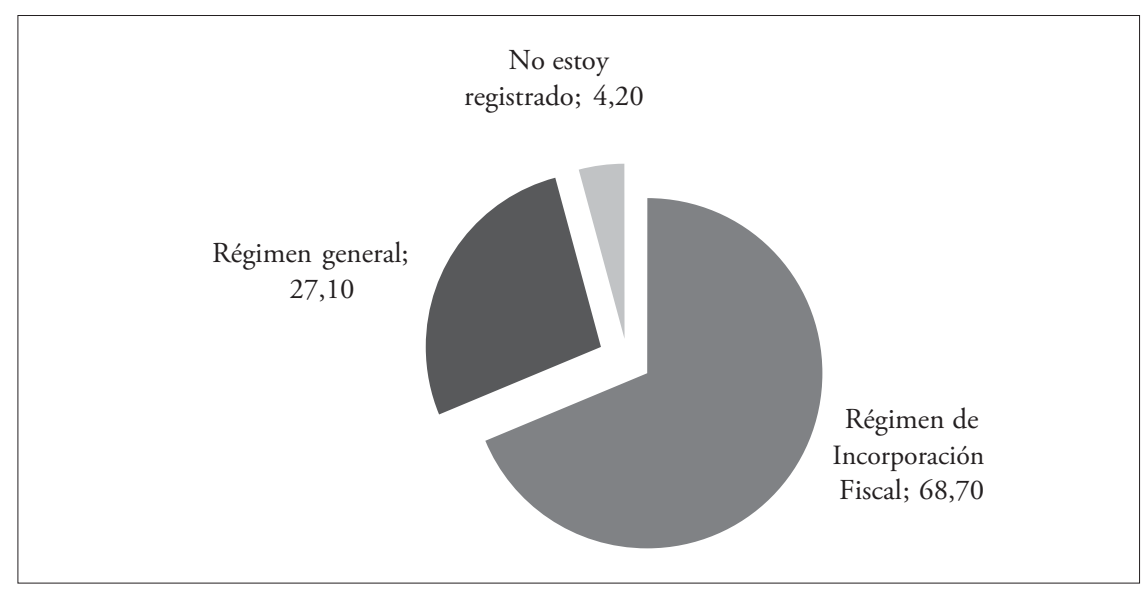

Fuente: diseńo propio a partir de la aplicación del cuestionario.

Gráfica 3. Planeación del pago de sus impuestos.

En la investigación realizada, se encontró que el 95,70\% de los empresarios que se les aplicó el cuestionario, llevan a cabo la planeación del pago de sus impuestos, además de apoyarse en los servicios de profesionales externos de la materia. El 4,30\% no lleva a 
Las mipymes mexicanas y el régimen de incorporación fiscal: un análisis de la reforma fiscal 2014 / López, et. al.

cabo dicha planeación; manifestaron que realizan el cumplimiento de sus obligaciones fiscales cuando la fecha de presentación de las mismas está próxima a llegar.

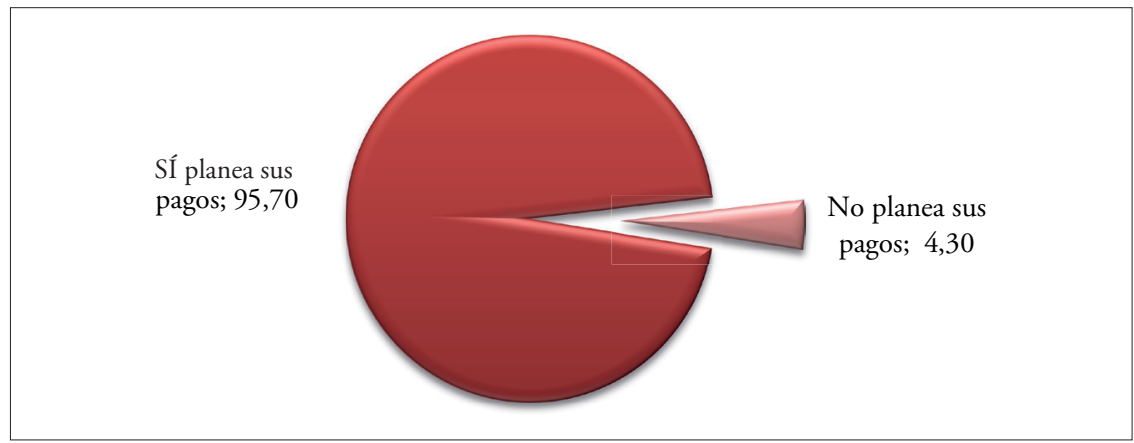

Fuente: diseńo propio a partir de la aplicación del cuestionario.

Gráfica 4. Está de acuerdo con la medida del gobierno.

La mayoría de los empresarios que respondieron a los cuestionamientos de esta investigación, manifestaron que este nuevo régimen no les afecta $(58,30 \%)$ ya que es muy práctico su cumplimiento con la participación de las programas electrónicos que proporcionan las autoridades fiscales, sin embargo, el 12,60\% y el 29,20\% manifiestan su inconformidad sobre esta medida, ya que consideran que son los mismos contribuyentes cautivos, los que cumplen con sus obligaciones fiscales, a quienes les afecta esta medida.

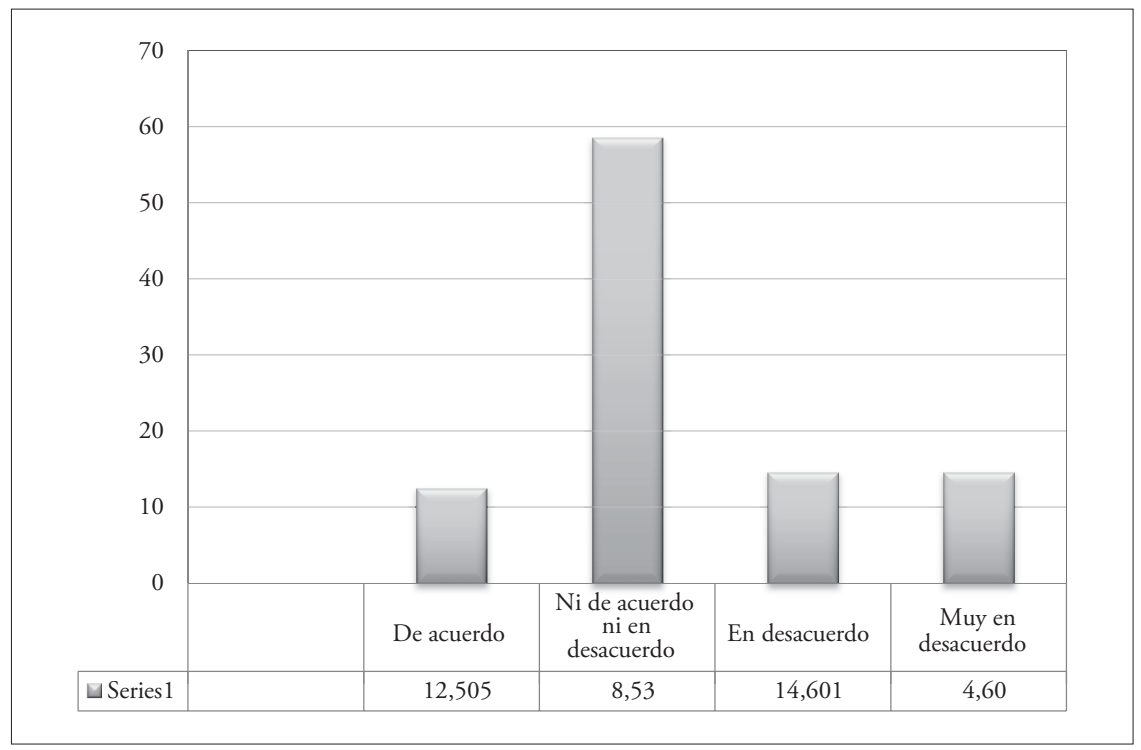

Fuente: diseńo propio a partir de la aplicación del cuestionario. 
Finalmente, como resultado de la aplicación de instrumentos de investigación, también se llega a la conclusión de que se ubica al empresario como micro y pequeño empresario por tratarse de empresas casi de índole familiar, como se señala anteriormente, y que por sus ingresos y movimientos se encuentran tributando mayormente en RIF (68,7\%), y solo el $27,1 \%$ en actividad empresarial del régimen general, por así convenir a su control fiscal, administrativo y contable.

Gráfica 5. Información contable del empresario.

Respecto a las obligaciones fiscales que cumplen este tipo de empresas en su régimen, cuentan con el apoyo externo de profesionales de la contaduría pública en un $83 \%$, lo que les permite cumplir en tiempo y en forma con dichas obligaciones, además de llevar un control de sus movimientos empresariales a través de registros contables como son: bancos, solo el 6,5\%, sumado a un registro contable formal $(93,5 \%)$.

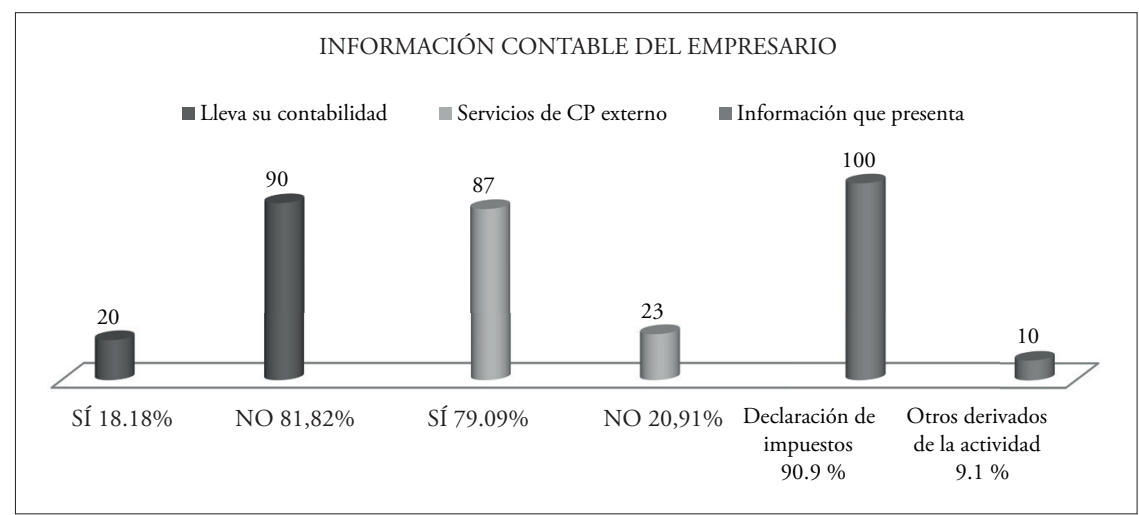

Fuente: diseño propio a partir de la aplicación del cuestionario.

Gráfica 6. La información que proporciona el SAT y las agrupaciones empresariales ha sido de utilidad para tu empresa.

También los representantes de las agrupaciones empresariales han jugado un papel importante para que los empresarios se familiaricen con la nueva reforma fiscal, ya que imparten cursos de información y actualización fiscal enfocándose a proporcionar las herramientas a los empresarios para que lleven a cabo el cumplimento de sus obligaciones con apego a las disposiciones que marcan las distintas leyes que rigen a las empresas. En el caso de la CANACO, esta se coordina con el SAT para llevar a cabo dichos cursos, que sirven para que los empresarios puedan contar con información necesaria y suficiente. Así mismo la CANIRAC proporciona orientación acerca de la administración de negocios y en ocasiones se imparten cursos en coordinación con 
Las mipymes mexicanas y el régimen de incorporación fiscal: un análisis de la reforma fiscal 2014 /

López, et. al.

los colegios de profesionales de la contaduría y el SAT, mismos que se imparten a los socios de ese organismo empresarial.

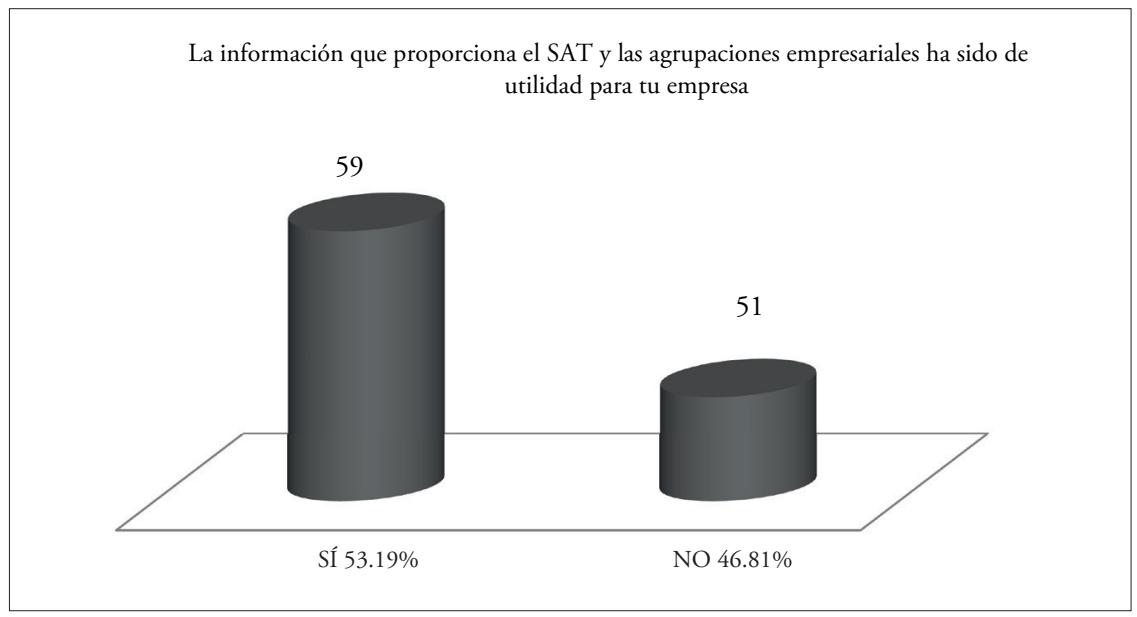

Fuente: diseño propio a partir de la aplicación del cuestionario.

Lo anteriormente expuesto da respuesta a un cuestionamiento: ¿cuáles son las repercusiones de la nueva reforma fiscal para las micros y pequeñas empresas del sector comercial, industrial y de servicios? Como se explica en este apartado, las repercusiones de la imposición fiscal para los empresarios registrados en RIF.

\section{Conclusión}

En lo referente a lo que implicó el cambio de régimen fiscal de las mipymes de ser un REPECO o régimen intermedio, se detectó que es muy complejo, por lo que la reforma fiscal integral deberá contemplar una inyección de simplicidad en el cumplimento de sus obligaciones fiscales. Se deberá además considerar la eliminación de varios tratamientos especiales a diversos sectores de la economía, pues se ha visto que hay mexicanos que pagan muchos impuestos, mientras que otros pagan muy poco o no pagan nada.

Lo anterior implica que la evasión fiscal sea muy alta, por lo que se deben hacer esfuerzos para reducirla, lo que incluye acciones de simplificación administrativa, creación de estímulos para incrementar la cultura de pago, y aplicación estricta de la ley. Asimismo, las autoridades fiscales establecen altas repercusiones financieras a los contribuyentes que permanecen en la informalidad o incumplen sus obligaciones fiscales. En este tenor, existe todavía corrupción en el brazo recolector del gobierno, práctica que debe ser atacada con mayor firmeza. El comercio informal sí le cuesta 
a los ciudadanos mexicanos, independientemente de que es un problema serio de legalidad y equidad en la aplicación de la ley de manera homogénea a todos los sectores de la población.

Dentro de los objetivos de la nueva reforma fiscal, también está el combatir la informalidad, por lo que la implementación de dicho acontecimiento originó un incremento en el padrón de contribuyentes del SAT; para llevar a cabo la confrontación de los resultados de la investigación, se consultaron los informes que emite la SHCP en forma periódica, en los cuales se detectó un aumento en el padrón de contribuyentes, cumpliendo con esto uno de los objetivos de la iniciativa de ley, entre ellos la regularización de los contribuyentes en su situación fiscal, de igual manera las autoridades fiscales han combatido la informalidad enviando invitaciones a los contribuyentes a regularizar su situación fiscal, tanto en el pago de sus impuestos así como en la situación administrativa.

El empresario tiene que saber que su dinero se gasta bien y de manera eficiente. Es decir, el incentivo más importante para fomentar el pago de impuestos es gastar bien. Los contribuyentes deben poder apreciar que sus impuestos sirven para mejorar el nivel de vida de todos los mexicanos. Conviene aquí retomar dos conceptos esenciales: el de la transparencia, que implica claridad en la asignación y uso de los recursos públicos; y el de la rendición de cuentas, que sugiere que para cumplir con el deber ciudadano del pago de los impuestos, debe abatirse la impunidad.

El cambio acelerado de la economía del país al cual nos estamos enfrentando y al mismo tiempo el de las disposiciones fiscales que reglamentan las transacciones del ente económico, nos llevan a estar continuamente actualizados para aprender y aplicar las herramientas fiscales y la interpretación adecuada de las misma, para la mejor toma de decisiones fiscales, propiciando el desarrollo de las empresas.

Por lo tanto, al entrar en vigor la nueva reforma fiscal 2014, la reacción de los contribuyentes del sector empresarial es aplicar estrategias para el cumplimiento de sus obligaciones en materia fiscal y administrativa, en tiempos y formas.

\section{Bibliografía}

Araújo, Arcelia (2008). "Política fiscal: golpe mortal a las mipymes". A Fondo Análisis y Expresión, No. 60, p. 22, México.

Chandler, D. Alfred (2000). La relación entre la estrategia y la estructura: un estudio de la coherencia en el contexto español, en decisiones estratégicas. Coordinadores de la obra: De Galán Zazo, José Ignacio y Sánchez Bueno, María José. México, Editorial Macmillan Publishers S.A. de C.V. 
Las mipymes mexicanas y el régimen de incorporación fiscal: un análisis de la reforma fiscal 2014 /

López, et. al.

De Soto, Hernando (1989). The other path: the invisible revolution in the third world. HarperCollins / El otro sendero: La revolución invisible en el Tercer Mundo. EUA, Editorial HarperCollins.

DOF - Diario Oficial de la Federación (2014). Publicación de reforma de la Ley del Impuesto sobre la Renta, 18 de diciembre de 2013.

Publicado el 18 de enero de 2012, en donde se modifica la Ley para el Desarrollo de la Competitividad de la Micro, Pequeńa y Mediana Empresa. (LDCMPME), consultado el 20 de octubre de 2014.

Ernst \& Young (2014). Exposición de motivos de la reforma fiscal 2014. En: <<http:// www.ey.com/MX/es/Services/Tax/Tax_alert_-propuesta_de_reformas_fiscales_20 14>>, consultado el 14 de julio de 2014.

Fuentes, A. (2014). Análisis y comentarios al nuevo Régimen de Incorporación Fiscal. En: <<http://www.ecacontadores.mx/content/analisis-rif >>. Fuente: Exposición de motivos de la iniciativa de Ley de Impuesto sobre la Renta para 2014, consultado el 20 de julio de 2014.

INEGI (2014). DENUE - Directorio Estadístico Nacional de Unidades Económicas. En: <<http://www3.inegi.org.mx/sistemas/mapa/denue/default.aspx>> consultado el 23 de junio de 2014.

LDCMPME - Ley para el Desarrollo de la Competitividad de la Micro, Pequeńa y Mediana Empresa.

Loayza, N.V. (1996). The economics of the informal sector: A simple model and some empirical evidence from Latin America, Policy Research Working Paper 1727, Washington, DC, WorldBank, EUA.

México emprende (2011). Pymes, Nuestras Empresas, volumen II, número 6. En: <<http://www.contactopyme.gob.mx/archivos/boletin/boletinII-6.pdf〉> consultado el 12 abril de 2012

OMC (2009). La globalización y el empleo en el sector informal en los países en desarrollo. Estudio conjunto de la Oficina Internacional de trabajo (OIT) y la Secretaría de la Organización Mundial del Comercio (OMC), Editorial: publicaciones de la OMC, Suiza.

Rodríguez, Marco Antonio (2010). Métodos de investigación. Diseño de proyectos y desarrollo de tesis en ciencias administrativas, organizacionales y sociales. Capítulo IV. Formulación de proyectos de investigación, editorial UAS, Serie: textos académicos, México.

Santesmases, Miguel (2010). DYANE versión 4, Diseño y Análisis de encuestas en investigación social y de mercados, primera edición, España. 
SIEM (2014). Información Estadística SIEM. En:

<<http://www.siem.gob.mx/siem/estadisticas/BrutoXedo.asp?p=1 >> consultado el día 20 de abril de 2014.

\section{Cómo citar este artículo:}

López, M.; González, M. y Rivera, M. (2014). "Las mipymes mexicanas y el régimen de incorporación fiscal: un análisis de la reforma fiscal 2014", Oikos No 38, 63-81, Escuela de Administración y Economía, Universidad Católica Silva Henríquez (UCSH), Santiago de Chile.

[http://ediciones.ucsh.cl/revistas.php]

Fecha de recepción: 02 / 10 / 2014

Fecha de aceptación: 26 / 01 / 2015 
